\title{
HORIZONTAL ACCURACY ASSESSMENT OF GOOGLE EARTH DATA OVER TYPICAL REGIONS OF ASIA
}

\author{
Jing Guo ${ }^{1}$, Jixian Zhang ${ }^{1}$, Haitao Zhao ${ }^{1}$, Chang Li $^{1}$, Jin Zhou ${ }^{1}$, Hongjing Tu${ }^{1}$, Ying Zhao ${ }^{1}$ \\ ${ }^{1}$ National Quality Inspection and Testing Center for Surveying and Mapping Products, Beijing, China - gj_season@163.com
}

Commission III, ICWG III/IVb

KEY WORDS: Google Earth, Horizontal Accuracy Assessment, Remote Sensing

\begin{abstract}
:
Google Earth provide the most accurate and available global high resolution imagery, covering nearly the entire land surface of the earth. However, the precision of Google Earth's data has not been fully validated.The traditional ground measurement method is difficult to verify the horizontal precision of remote sensing over a large area. This paper focuses on typical regions of Asia, aiming to verify the precision of GE's data based on purchased WorldView (WV) data by utilization of statistical analysis method.The results show that the highest precision has been estimated as 4.96-6.83 meters over the part of Japan, India and Kazakhstan, respectively. The lowest precision 16.53 and 16.59 meters primarily appear mountainous terrain, including the part of Israel and Syria.The result also presents the horizontal precision estimated in Japan, India and Kazakhstan, which is slightly higher than the precision estimated in Israel and Syria. The regions with larger deviation of relative errors have apparent influence on horizontal accuracy assessment of GE's imagery. Accuracy assessment may be affected by terrain features and the insignificant feature points over the study area. The results suggest that the most of horizontal accuracy of GE's high resolution imagery over the most of study regions fulfills precision requirement of 1:50000 maps.
\end{abstract}

\section{INTRODUCTION}

Google Earth (GE) is the most popular virtual globe that offers free access to high resolution imagery for most of the planet. Since the launch of the program in 2005 by Google, it has been downloaded more than 1 billion times to desktop and mobile clients (CUTBERTO,2016; GOOGLE, 2011). GE is the most accurate and available global high resolution imagery data, cover nearly the entire land surface of the earth. Researchers always focus on assessing the accuracy of GE data since it launched. The data used for precision verification mainly come from high precision remote sensing and field measurement.The precision of GE image is evaluated by using high precision remote sensing image or same precision remote sensing image, and their position RMS errors is counted.

The most common precision checking method is to verify DOM accuracy with field data, because it comes from highprecision ground control points. However, due to the large amount of field measurement, it is difficult to obtain a large area and a large number of measurement points, which limits its large-scale application. The spatial resolution of the WorldView panchromatic image is $0.31 \mathrm{~m}$, which is the highest spatial resolution of commercial remote sensing satellite in the world. Its precision assessment has been carried out by many researchers. The research results show that wv data has high positioning accuracy, and it is feasible to use wV data to evaluate GE precision results.

Horizontal accuracy evaluation of GE's imagery have been studied by many researchers and many results have been obtained around the world, which shows the accuracy quality is certain regional differences. The precision of the data has not been fully validated (Becek and Ibrahim, 2011). According to the related studies at home and abroad, the control points extracted from the different datasets are used to evaluate the positioning accuracy of GE's data, and estimated a global horizontal root mean squared error in the range from 30 to 50 meters. The study also found that by comparing the control points with the 1: 50,000 topographic map, the amplitude of the error will change with the location, and the maximum error can reach hundreds of meters. CUTBERTO suggests that GE's high resolution imagery meets the horizontal accuracy requirements of the ASPRS for the production of "Class 1" 1:20,000 maps; however, his research findings also show that georegistration and large horizontal errors occur in GE's imagery.

Research shows that data precision evaluation conclusions on a global scale are difficult to apply to all regions. However, current studies tend to converge on middle and small scale areas or typically morphologic regions. And the traditional ground measurement method is difficult to verify the horizontal precision of remote sensing over a large area. Therefore, it is of great significance to use wv data to carry out large-area GE data location accuracy assessment in a typical region of Asia. Based on the previous research results and with the spatial distribution in consideration(FLANAGIN;MERZGER, 2008), it selects the plain, hilly, basin and mountain terrains covered in Japan, India, Kazakhstan, Israel and Syria as typical study areas.

This paper aims conduct a comprehensive assessment for the horizontal accuracy of GE' imagery, which contains high, medium and low-resolution data and its resolution distribution is not uniform in the global scope. In this paper, high-resolution data (pixel size of less than $1 \mathrm{~m}$ ) are selected for horizontal precision evaluation. The reference data for this article is derived from the purchased WV data. This paper uses statistical analysis methods to evaluate the accuracy of GE remote sensing images in typical Asian regions using vw , and studies the relationship between GE accuracy distribution and terrain features. 


\section{DATA AND STUDY AREAS}

\subsection{Data}

Google Earth (GE) is a virtual globe software developed by Google. It arranges satellite photos, aerial photographs and GIS on a three-dimensional model of the earth. The imagery of GE is not a single data source, but satellite imagery Integration with aerial photography data, it integrates WorldView, Quickbird, Landsat and SPOT5 in one. Google claims that Quickbird imagery has a circular error at the $90 \%$ confidence interval CE90 (Circular Error 90\% ) of 23 and and that WorldView-1 and 2 have a CE90 of $5 \mathrm{~m}$ (CUTBERTO,2016).

The WorldView satellite has an orbital height of $617 \mathrm{~km}$, a panchromatic band of $2 \mathrm{~m}$, another eight $1.24 \mathrm{~m}$ multispectral bands, and eight $3.7 \mathrm{~m}$ shortwave infrared beams. World Vision is a pendulum-scan imaging. The imaging capability is greater than $20^{\circ}$ in side swing, the width of the sub-satellite point is $13.1 \mathrm{~km}$, and the imageable area is $6.8 \times 105 \mathrm{~km} 2$ per day. In order to evaluate the accuracy more accurately, the WV data was firstly orthorectified, and the data used for orthorectification is from SRTM data.

For the evaluation of horizontal accuracy in GE's data, this paper use WV's data for reference. We make contrastive analysis about the absolute positioning accuracy and relative positioning accuracy. Its analysis further clarified the reasons for the error by analyzing the errors along the rail direction and the vertical rail direction.

\subsection{Study Areas}

In this work, the study area covers the eastern, central and western Asia. The typical region of Asia is an important study region for accuracy assessment of GE, which exert a strong anthropogenic effect and complex terrain, because of its dense populations and rapid growth of its economy. In this paper, typical regions rather than the whole Asia are selected for analysis in order to identify more accurate accuracy assessment The study areas include Japan, India, Kazakhstan, Israel and Syria separately. The spatial distribution of the study area is shown in Figure 1. Japan, Parts of India and Israel are the most developed regions in study areas, which are densely populated and economically developed. Most of Israel and Syria are in desert areas with few ground objects. Especially in Syria where is suffer from the war in recent years. Kazakhstan is dominated by plains and is the largest landlocked country in the world, with a unique geographical environment. The above countries and region are selected to assess absolute positioning accuracy for GE's data using WV.

\section{METHODOLOGY}

\subsection{Accuracy Assessment Indicators}

In this study, we will analyze the horizontal accuracy assessment results of GE's imagery in terms of absolute and relative positioning accuracy. Absolute accuracy is measured by Root-Mean-Square Error, which represents the total error (including systematic error and random error). The absolute accuracy assessment indicators used in this study can be calculated by Eq 1 through Eq 3. Relative positioning accuracy is an important index to evaluate the relative geometric positioning accuracy of high-resolution remote sensing. It is a statistic that measures the relative error between checkpoints on an image. The purpose of evaluating the relative positioning accuracy of images is to avoid images with linear systematic errors in the absolute positioning errors of the images, which directly reflects the magnitude of random errors of imaging at different positions in the area. The common statistical indicators and statistical test such as RMSE, RMSEx, RMSEy and Relative Error Statistics(RES), respectively are considered. This paper makes a comparative study of various statistical indexes in the study area, and analyzes the horizontal position accuracy and characteristics of the GE data in study area. The results of these accuracy assessments are presented in the next section.

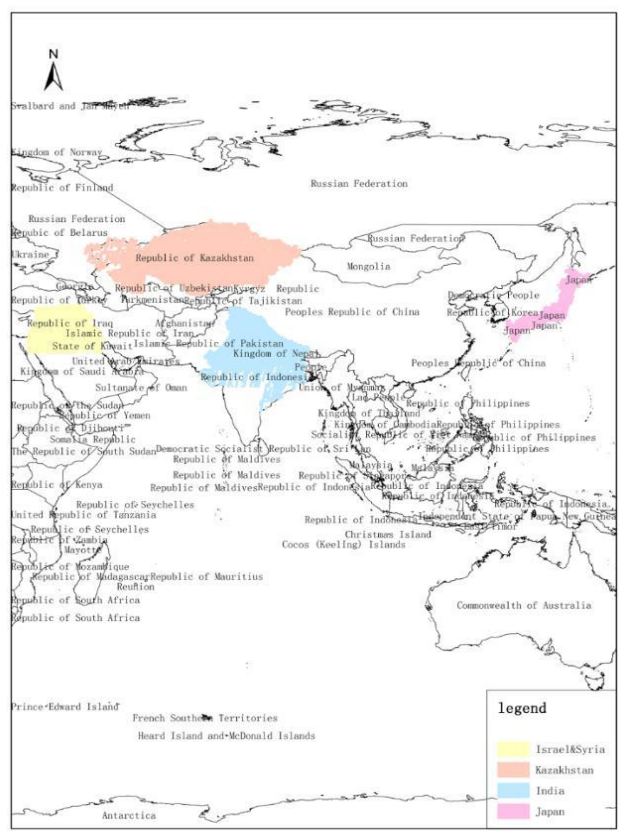

Figure 1. Study areas and the location of horizontal accuracy assessment of Google Earth data

The absolute accuracy assessment is represented using following equation:

$$
\begin{aligned}
& \operatorname{RMSE}_{x}=\sqrt{\frac{\sum_{i=1}^{N}\left(\mathrm{X}_{i}^{0}-\mathrm{X}_{i}\right)^{2}}{N}} \\
& \operatorname{RMSE}_{y}=\sqrt{\frac{\sum_{i=1}^{N}\left(Y_{i}^{0}-Y_{i}\right)^{2}}{N}}
\end{aligned}
$$

$$
R M S E_{x y}=\sqrt{\frac{\sum_{i=1}^{N}\left(\mathrm{X}_{i}^{0}-X_{i}\right)^{2}+\sum_{i=1}^{N}\left(Y_{i}^{0}-Y_{i}\right)^{2}}{N}}
$$

Where $\mathrm{N}$ is the number of observations $\mathrm{Xi}, \mathrm{Yi}$ are the assumed truth value $i$ is GE's imagery at poin

The absolute accuracy assessment can be calculated by:

$$
A_{i}=\sqrt{\left(\mathrm{X}_{\mathrm{N}}-\mathrm{X}_{M}\right)^{2}+\left(\mathrm{Y}_{N}-\mathrm{Y}_{M}\right)^{2}}
$$




$$
\begin{gathered}
B_{i}=\sqrt{\left(\mathrm{X}_{\mathrm{N}}^{\prime}-\mathrm{X}_{M}^{\prime}\right)^{2}+\left(\mathrm{Y}_{N}^{\prime}-\mathrm{Y}_{M}^{\prime}\right)^{2}} \\
C_{i}=\left|A_{i}-B_{i}\right| \\
\frac{1}{m} \sum_{i=1}^{m} \overline{C_{i}}
\end{gathered}
$$

Where $\quad \mathrm{X}_{\mathrm{N}}, \mathrm{Y}_{\mathrm{M}}$ is the assumed truth value at pint $\mathrm{M}, \mathrm{N}$

$\mathrm{X}^{\prime}{ }_{\mathrm{N}}, \mathrm{Y}^{\prime}{ }_{\mathrm{M}}$ is the GE's imagery at point $\mathrm{M}, \mathrm{N}$

$\mathrm{Ci}$ is the distances between any two points $\mathrm{M}$ is statistical results

\subsection{Assessment Procedure}

In order to evaluate the satellite geometric positioning accuracy, images acquired at different times in different regions of the world need to be collected and used as reference data.Based on the reference image, the corresponding feature points are collected on each evaluated image, and the coordinates of the detected point on the evaluated image and the corresponding control coordinates are used to evaluate the absolute positioning accuracy and relative positioning accuracy through the above calculation formula.The following four steps are required :

(1) Prepare the image to be detected and the reference image;

(2) Automatic matching or manual selection of checkpoints;

(3) Gross error elimination;
(4) Precision statistics.

Use the wv's image as reference data to match the inspection points uniformly on the image to be inspected. Use the rational polynomial model to back-project the coordinates of the checkpoint onto the image to obtain its corresponding image point coordinates. The calculated image point coordinates are compared with the actual matched image point coordinates, and the error of mean squares in the vertical direction (x), the error in the track direction (y) and the error in the plane are counted. Finally, calculate the average value of the result of the evaluation image.

\section{RESULT}

In this section the results of horizontal accuracy for GE's imagery will be presented. The analysis is based on the results of the data accuracy assessment, which is the result of comparing statistics between GE data and WV data. Horizontal accuracy assessment was computed more than 800 points measured on every study region. Statistics for horizontal accuracy was computed according to the above statistical indicators.

The results show that the highest precision has been estimated as 3.41-4.72 meters over the part of Japan, India and Kazakhstan, respectively. The higher precision (4.96-6.83) meters are mainly focus on India, Israel and Syria, respectively. In contrast, the lowest precision 16.53 and 16.59 meters primarily appear mountainous terrain, including the part of

\begin{tabular}{|c|c|c|c|c|c|}
\hline Study Area & $\begin{array}{l}\text { Absolute } \\
\text { Positioning } \\
\text { Accuracy }\end{array}$ & $\begin{array}{c}\text { Relative } \\
\text { Positioning } \\
\text { Accuracy } \\
\text { (along track) }\end{array}$ & $\begin{array}{c}\text { Relative } \\
\text { Positioning } \\
\text { Accuracy } \\
\text { (vertica track) }\end{array}$ & $\begin{array}{c}\text { Absolute } \\
\text { Positioning } \\
\text { Accuracy } \\
\text { (along track) }\end{array}$ & $\begin{array}{c}\text { Absolute } \\
\text { Positioning } \\
\text { Accuracy } \\
\text { (vertica track) }\end{array}$ \\
\hline \multirow{3}{*}{ Japan } & 4.96 & 2.88 & 1.19 & -6.89 & 0.36 \\
\hline & 9.27 & 2.73 & 7.67 & -0.81 & 17.36 \\
\hline & 4.72 & 1.35 & 1.32 & 6.26 & -5.18 \\
\hline \multirow{8}{*}{ India } & 5.01 & 1.61 & 2.25 & -4.39 & 5.01 \\
\hline & 6.83 & 2.89 & 2.11 & 12.10 & 6.84 \\
\hline & 6.01 & 1.57 & 1.21 & 10.45 & 5.58 \\
\hline & 4.51 & 1.35 & 2.43 & -6.12 & 1.84 \\
\hline & 3.75 & 1.16 & 2.63 & 2.77 & 1.81 \\
\hline & 5.42 & 1.78 & 1.79 & 9.23 & -0.03 \\
\hline & 6.72 & 3.18 & 2.82 & 7.11 & 10.22 \\
\hline & 5.22 & 3.45 & 5.54 & 0.38 & 2.94 \\
\hline \multirow{3}{*}{ Kazakhstan } & 7.04 & 1.38 & 1.32 & 5.28 & 14.12 \\
\hline & 3.41 & 0.98 & 0.97 & 2.77 & 1.58 \\
\hline & 3.95 & 2.27 & 2.09 & -1.76 & 3.48 \\
\hline \multirow{9}{*}{ Israel and Syria } & 5.17 & 1.69 & 1.70 & -0.23 & -0.14 \\
\hline & 6.10 & 3.63 & 6.789 & -1.84 & -7.87 \\
\hline & 4.98 & 2.43 & 2.72 & 7.43 & -2.21 \\
\hline & 5.08 & 3.58 & 3.32 & -2.27 & -6.62 \\
\hline & 3.45 & 1.87 & 1.870 & -0.51 & -0.30 \\
\hline & 16.59 & 2.77 & 2.26 & -19.96 & 40.74 \\
\hline & 4.00 & 1.09 & 0.75 & -2.57 & 4.61 \\
\hline & 6.76 & 4.48 & 6.22 & 2.58 & -3.76 \\
\hline & 16.54 & 0.90 & 4.08 & 44.15 & -0.44 \\
\hline
\end{tabular}
Israel and Syria. In conclusion, higher precision occur in plain and hill rather than mountain land, as expected.

Table 1. Error statistics of the accuracy assessment over typical regions of Asia

\subsection{Regional Accuracy Analysis}


This section presents the accuracy assessment results of GE data for each study area. To better understand the spatial distribution of precision of GE data, we analyze absolute and relative position accuracy over above representative regions. The error statistics of the accuracy assessment of every study area shown in Table 1.

In Japan, the highest precision has been estimate as 4.72 meters RMSE (RESx: 1.35, RESy: 1.32 pixels) in the eastern Japan. The lowest precision is estimated in 9.3 meters RMSE\{RESx: 2.73, RESy: 7.67 pixels\}. The larger horizontal errors are observed possibly due to lowest relative positional accuracy. We also found the vertical track direction of horizontal errors is significantly larger than the along track direction in GE's imagery in north of Japan (RMSEx:-0.81, RMSEy: 17.4 pixels) It indicates deviation of location accuracy possibly caused by the geometric distortion of mountainous regions of GE's image. In India, the highest precision has been calculated in 3.75 meters RMSE (RESx: 1.16, RESy: 2.63 pixels), the lowest precision is 6.83 meters RMSE (RESx: 2.9, RESy: 2.11 pixels). It has high absolute positioning accuracy and relative positioning accuracy. Kazakhstan's assessment results are similar to those of India, which showed the highest precision has been calculated in is 3.41 meters RMSE (RESx: 0.98, RESy: 0.97 pixels), the lowest precision is 7.03 meter RMSE (RESx: 1. 38, RESy: 1.32 pixels). In this assessment, absolute and relative position accuracy of GE's imagery are better than those from other study areas, which indicating that GE's data does not have significant bias above two regions mentioned. Furthermore, we have found that the difference between RMSEx and RMSEy are not significant. In Israel and Syria, the highest precision has been estimate as 3.45 meter RMSE (RESx: 1.87, RESy: 1.87 pixels) in the north of Israel. The lowest precision is estimated in 16.59 meter RMSE (RESx: 2.77, RESy: 2.62 pixels). The larger horizontal errors are observed possibly due to lowest relative positional accuracy. We also found the along track direction of horizontal errors is significantly lower than the vertical track direction in GE's data in south of Syria (RMSEx:-19.96, RMSEy:40.74 pixels ). The absolute accuracy variation over study area is present in Figure 2.

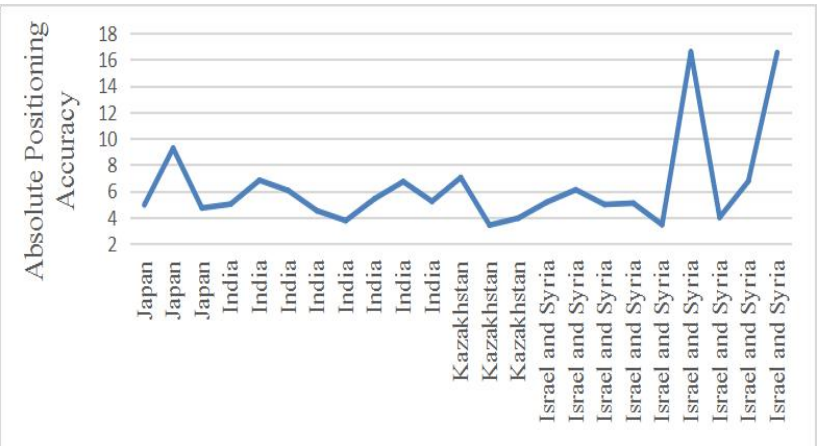

Figure 2. Absolute accuracy variation of GE's data over Japan,India,Kazakhstan and Israel and Syria

We found that the absolute positioning accuracy deviations in the along track direction and the vertical track direction are very large, and the error in the vertical track direction is significantly greater than the along track direction error, up to 40.7, the overall deviation of the area in the along track direction. In addition, there is not remarkable correlation between the absolute position accuracy and latitude Over study areas.

\subsection{Accuracy Analysis for Terrain}

Absolute positioning accuracy distribution on different terrains are shown in Figure 3. In this histogram, the blue represents the plain terrain, yellow represents the hill terrain and red represents the mountain terrain. The results show that horizontal accuracy in plains are higher than that of the hilly and mountainous areas. The precision ranging from 4.96-6.83 meters mostly appear in India and Kazakhstan. Additionally, the highest precision is observed in 4.51 meters in the plains of India. As we can see from the chart, the precision in mountain terrains is significantly lower than that in the plain and hilly terrains.

The lowest precision occurs in Syria and Israel, at 16.53 and 16.59 meters, respectively, which is also the lowest accuracy of the entire study area. The higher precision is observed in hilly terrains, including Japan and India, except individual areas. The results show that the accuracy assessment results of Japan, India and Kazakhstan in different terrain features are relatively good, and the accuracy assessment results of Israel and Syria are significantly affected by the terrain features.

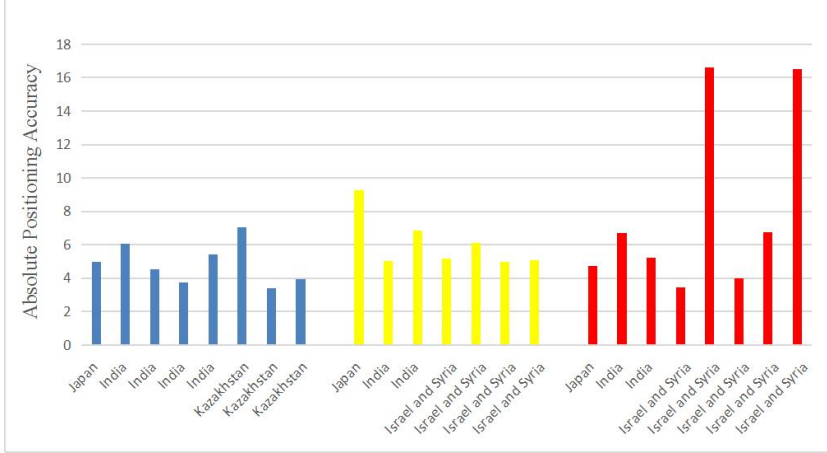

Figure 3. Absolute positioning accuracy distribution on plains (blue columns ), hills (yellow columns), and mountain (red columns) terrain

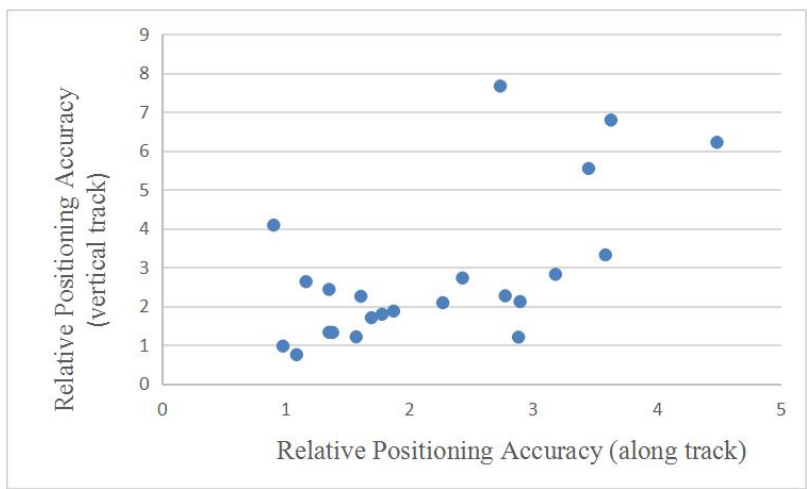

Figure 4. Spatial distribution of relative positioning accuracy in GE's imagery

\subsection{Comparison of absolute and relative position accuracy}

As shown in Figure.4, the spatial distribution of relative position accuracy is analyzed in GE data over the study areas. 
As can be seen from the chart, relative positioning accuracy of most research areas is concentrated within the interval of 4 pixels, indicating that the distortion of the GE image does not exceed 4 pixels. The relative positioning accuracy of a few research areas is in the range of 6-8 pixels, indicating that the GE image in this area has large distortion. It demonstrates that there is no obvious the distortion of CCD satellite images over India, Kazakhstan, parts of Japan and parts of Israel and Syria.

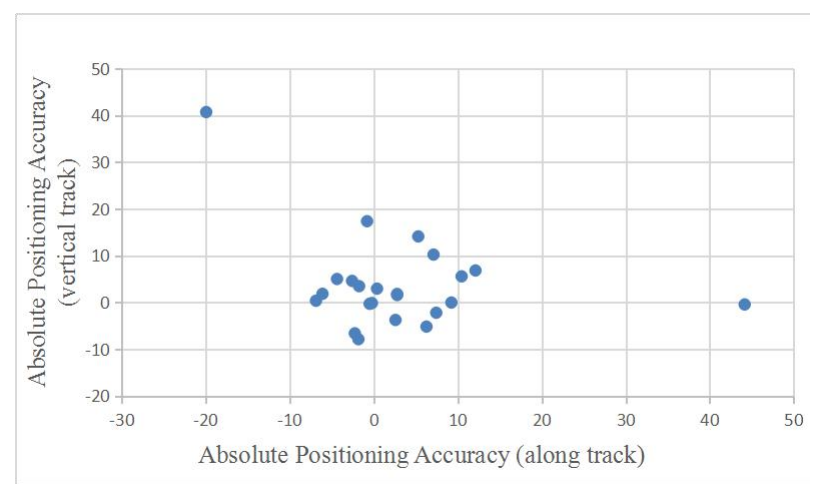

Figure 5. Spatial distribution of absolute positioning accuracy in GE's imagery

For low absolute positioning results, through the analysis of absolute positioning accuracy, it is directly affected by the absolute positioning accuracy of the vertical rail and along the rail. As the previous analysis of the Syrian area of Israel, the lowest absolute accuracy is $16.59 \mathrm{~m}$, and the relative positioning accuracy is 2.77 pixels RESx and 2.26 pixels RESy, but the large deviations are found to be the absolute accuracy on the vertical rail and along the rail, respectively -19.96 pixels RMSEx and 40.74 pixels RMSEy over Israel and Syria, especially larger deviations in the vertical rail direction were found. Spatial distribution of absolute positioning accuracy is shown in Figure 5. Comparing its relative accuracy, it can be found that the deviation of the positioning accuracy is probably due to the insignificant feature points and the single feature type for Syrian region of Israel,which leads to the deviation of the matching results.

Similarly, in Japan, the worst absolute positioning accuracy is $9.26 \mathrm{~m}$, and the positioning accuracy has a large offset of 7.67 pixels in the vertical track direction, but the absolute positioning accuracy has an offset of up to 17.38 pixels in the along-track direction. This may be caused by the internal distortion and the external cause of the image. Although our previous analysis believes that the accuracy result is greatly affected by the terrain, there is also the possibility of deviations in checkpoint matching due to unclear local images or unclear feature points. Studies have shown that the accuracy assessment meet the accuracy requirements of the 1: 50000 scale results for most areas. However, we suggest to prefer the plain and hilly terrain in the GE data, and the areas with obvious features, and ensure that the GE data used is highresolution remote sensing images.

\section{CONCLUSION}

We performed analysis to investigate the absolute and relative position accuracy in Japan, India, Kazakhstan, Israel and Syria separately. The result presents the horizontal precision estimated in Japan, India and Kazakhstan, which is slightly higher than the precision estimated in Israel and Syria. Similarly, systematic errors are not apparent in GE's imagery in most of the study regions. The positioning accuracy deviation may be related to the distortion of CCD satellite images for Japan.On the one hand, the positioning accuracy deviations in Israel and Syria may be affected by terrain features, on the other hand, the matching results may be biased due to the insignificant feature points in the region and the single feature type.

Over study areas, there is not remarkable correlation between the absolute position accuracy and latitude; however, the regions with larger deviation of relative errors have apparent influence on horizontal accuracy assessment of GE's data.

Regarding the application of GE data in scientific research and engineering projects, the results suggest that the most of horizontal accuracy of GE's high resolution imagery over the most of study regions fulfills precision requirement of 1:50000 maps (maximum RMSE of 10 meters in plain and hill).

\section{ACKNOWLEDGEMENTS}

This work has been supported by Mapping Global Program. The authors also acknowledge the WorldView. for accessing available data from Google Earth .

\section{REFERENCES}

Becek, K., Ibrahim, K., On the positional accuracy of the Googleearth imagery. TS051-Spatial Information Processing Ipaper no.4947. In:FIG Working Week 2011,Marrakech,Morocco,18-22 May 2011.

Cutbertc., Uriel., HORIZONTAL., POSITIONAL ACCURACY OF GOOGLE EARTH' S IMAGERY OVER RURAL AREAS: A STUDY CASE IN TAMAULIPAS, MEXICO. BCG - Boletim de Ciências Geodésicas - On-Line version, ISSN 1982-2170 ,http://dx.doi.org/10.1590/S198221702013000400005 .

FLANAGIN, A.J., MERZGER, M.J., The credibility of volunteered geographic information.GeoJournal,72,137148,2008 .

Gesch DB, Oimoen MJ, Evans GA (2014) Accuracy assessment of the US Geological Survey National Elevation Dataset, and comparison with other large-area elevation datasets: SRTM and ASTER. US Geological Survey. 2331$12582331-1258$.

Google. Source for elevation data. Google Product Forums. 2008.Available.at:https://groups.google.com/forum/?fromgroup s=\#!topic/earthdata/KsRTsXULRNk.Accessed:20 March 2013.

Manual HS (2010) American association of state highway and transportation officials (AASHTO). Washington, DC 10. 23.

Monteys, X., Harris, P., Caloca, S., Cahalane, C., Spatial prediction of coastal bathymetry based on multispectral satellite imagery and multibeam data, Remote Sensing, vol. 7/issue 10, pp 13782-13806, 2015.

Potere D (2008) Horizontal positional accuracy of Google Earth's high-resolution imagery archive. Sensors 8: 7973-7981. https://doi.org/10.3390/s8127973 PMID: 27873970 
Yinsong, Wang., Yajie, Zou., Kristian, Henrickson., 2017:Google Earth elevation data extraction and accuracy assessment for transportation applications.PLOS ONE, 12(4).doi.org/10.1371/journal.pone.0175756.

Yu L, Gong P (2012) Google Earth as a virtual globe tool for Earth science applications at the global

scale: progress and perspectives. International Journal of Remote Sensing 33: 3966-3986.

Zou Y, Zhang Y, Lord D (2013) Application of finite mixture of negative binomial regression models with

varying weight parameters for vehicle crash data analysis. Accident Analysis \& Prevention 50: 1042- 1051. 